\title{
Evaluación de la concentración y competencia del sector bancario ecuatoriano en el periodo 2006-2018
}

\section{Evaluation of the concentration and competition of ecuadorian banking sector in 2006- 2018 period}

\author{
URL: http://revistas.uta.edu.ec/erevista/index.php/bcoyu/article/view/882 \\ DOI: http://dx.doi.org/10.31164/bcoyu.24.2020.882
}

Armando Romero - Galarza ${ }^{1}$, Gustavo Flores - Sánchez², Brigitte Reyes-Zambrano ${ }^{3}$, Jorge Campoverde - Campoverde ${ }^{4}$

Fecha de recepción: 27 de noviembre de 2019 . Fecha de aceptación:5 de marzo 2020

\begin{abstract}
Resumen
El presente trabajo se centra en la determinación del grado de concentración y competencia de mercado del sector bancario ecuatoriano; medido con información de las cuentas de capital, cartera de crédito, depósitos e inversión en el periodo 2006-2018. La investigación presenta una metodología de corte longitudinal, mediante la utilización de datos con una dimensión temporal y espacial; empleando diferentes índices de carácter estructural y no estructural. Los principales hallazgos indican que en el sector bancario ecuatoriano no existe concentración de mercado, además, los bancos tienen la capacidad de fijar sus precios, que deben oscilar entre los límites establecidos por los organismos de regulación como el Banco Central del Ecuador, el Ministerio de Finanzas y la Superintendencia de Bancos
\end{abstract}

Palabras clave: Índice, concentración de mercado, banco, poder de mercado

\begin{abstract}
The present work focuses on the determination of the degree of concentration and market competition of the Ecuadorian banking sector, measured whit the information of the accounts of capital, credit portfolio, deposits and investment in the period 2006-2018; The research presents a longitudinal-sectional methodology through the use of data with a temporal and spatial dimension; using different structural and non-structural indexes. The main findings indicate that in the Ecuadorianbanking sector there is no market concentration, in addition, that banks can set their prices, that should oscillate between the limits established by the agencies of regulation such as: The Central Bank of Ecuador, the Ministry of Finance and the Superintendence of Banks.
\end{abstract}

Keywords: Index, market concentration, bank, market power

\footnotetext{
${ }^{1}$ Universidad de Cuenca. Facultad de Ciencias Económicas y Administrativas. Grupo de Investigación Empresarial. Cuenca - Ecuador. E-mail: armando.romerog@ucuenca.edu.ec.ORCID: http://orcid.org/0000-0003-1149-4299

2 Universidad de Cuenca. Facultad de Ciencias Económicas y Administrativas. Grupo de Investigación Empresarial. Cuenca - Ecuador. E-mail gustavo.flores@ucuenca.edu.ec.ORCID: http://orcid.org/0000-0003-4123-2644.

3 Universidad de Cuenca. Facultad de Ciencias Económicas y Administrativas. Cuenca - Ecuador. E-mail: brigitte.reyes@ucuenca.edu.ec. ORCID. https://orcid.org/0000-0003-1572-2455

${ }^{4}$ Universidad de Cuenca. Facultad de Ciencias Económicas y Administrativas. Grupo de Investigación Empresarial. Cuenca - Ecuador. E-mail. jorge.campoverde@ucuenca.edu.ec. ORCID: http://orcid.org/0000-0002-1633-5644
} 


\section{Introducción}

Los diferentes mercados presentan características y situaciones que dificultan entender cómo actúan las organizaciones para enfrentar la competitividad existente en su entorno, de este modo cada institución mantiene una lucha constante con la competencia presente, la cual está acompañada del dinamismo del mundo actual que otorga grandes beneficios a aquellas empresas o firmas que pueden desarrollar tácticas que les permitan ser competitivas y sobrevivir en el mercado; debido a que un componente crucial es el saber establecer las mejores estrategias que satisfagan las necesidades de los clientes, quienes día a día presentan mayores exigencias ocasionadas por los avances tecnológicos y las facilidades o comodidades que estas puedan brindar.

La importancia de los diferentes grupos y organizaciones en un país, se ve reflejada en la participación que tienen dentro de su economía; así un sector primordial para el desarrollo de la misma es el financiero; el cual para autores como Fry, Levine, McKinnon, Shaw e instituciones como el Banco Mundial, según se cita en Morales, García, y Ángeles (2013), es considerado un factor esencial en el fomento del ahorro, asignación de recursos y crecimiento económico. En este contexto el sector financiero ecuatoriano representa uno de los pilares fundamentales, de manera específica el bancario, considerado uno de los más importantes debido a su aporte al Producto Interno Bruto (PIB) que representó 3'536.287 millones de USD en el año 2016 (BCE, 2018). Por otra parte, su importancia también radica en el desarrollo de sus actividades, las cuales entre otras se centran en la captación de ahorros proveniente de personas $\mathrm{u}$ organizaciones y distribuirlos entre quienes requieren créditos; además existe el papel del banco central, encargado de controlar y establecer los mecanismos dentro de los cuales se realizan las diferentes operaciones.

En el ámbito empresarial, Penrose (1958) considera la empresa como una organización dedicada a la obtención de beneficios monetarios mediante la venta de productos 0 servicios; la cual posee diferentes dinámicas que le permiten crecer y dominar el mercado en base a la venta de sus productos; definición contrastada y evidenciada por Vargas y Rodríguez (2016).

En este sentido, se observa que en las diferentes instituciones bancarias a nivel país, no existe un número fijo de bancos en el periodo considerado para el estudio; situación que se considera como una problemática para el sector, la cual puede deberse a la existencia de cárteles o dominio de mercado por parte de determinadas organizaciones. De esta forma, resulta primordial conocer la evolución de las instituciones y la estructura del sector; por ello el principal objetivo del presente estudio es: Realizar un análisis de concentración en el sistema bancario ecuatoriano y determinar la existencia de algún tipo de poder de mercado; además de los siguientes objetivos específicos: i) Obtener los grados de concentración de los bancos en diferentes periodos de tiempo y ii) Obtener un índice de concentración por grupos de entidades, bancos privados grandes y bancos públicos.

La investigación parte de la premisa conocida como Estructura-Conducta-Resultado (ECR), que surgió a inicios y hasta mediados de los años setenta, esta considera los elementos de la estructura empresarial industrial, el tamaño de las empresas, el grado de concentración de oferentes y demandantes, y la tecnología; teniendo como determinante de conducta las economías de escala. De igual manera, estas establecen el desempeño de la industria así como de los agentes que la conforman (Tarziján y Paredes, 2006). En lo referente al paradigma ECR, Edward Mason y Joe Bain, de la Escuela de Harvard, afirman que los mercados que presentan una alta concentración conducen a comportamientos empresariales y resultados que están en contra del bienestar, dicho de otro modo, no podrían ser diferenciados de los resultados obtenidos por un cártel formado por todas las firmas o las que tengan mayor tamaño; de esta manera, se deduce que estos índices brindan suficiente evidencia para establecer el nivel de competencia existente (Martínez, Zuleta, Misas, y Jaramillo, 2016).

El trabajo de investigación se encuentra estructurado de la siguiente manera; en el primer apartado se da a conocer el tema de estudio, el segundo incluye lineamientos teóricos que engloban la definición de términos, conceptos y revisión de literatura; en la tercera sección se presenta la descripción del objeto de indagación y de manera seguida se redacta la metodología aplicada. En la quinta sección se detalla el campo investigativo y los datos de análisis, posteriormente se describen los resultados o principales hallazgos; finalmente, se puntualizan las conclusiones derivadas de la investigación.

\section{Lineamientos teóricos}

En las diferentes economías a nivel mundial resulta fundamental realizar un análisis de la coyuntura económica mediante estudios o investigaciones de índole financiero, de manera que permitan conocer la evolución del sector y su influencia en los mercados, al igual que su importancia en el aporte y dinamización de la economía en cada país. En consecuencia, las instituciones financieras representan un rol importante entre los diferentes agentes económicos actuando como un pilar dinamizador de la economía mediante la generación de riqueza y productividad (Flor, 2013). Por otra parte, el estudio de este campo permite conocer el rol que desempeñan los diferentes actores del sistema y de manera específica el formado por la banca pública y privada.

Según BanEcuador (2018) el Sistema Financiero Nacional (SFN) está constituido por instituciones financieras que se encuentran reguladas por la Superintendencia de Bancos y por la Superintendencia de Economía Popular y Solidaria; mediante el cual se regularizan las diferentes operaciones, tales como: ahorro, inversiones, obtención de créditos, entre otras que realizan los clientes. En contraste, para Romero (2015) el sistema financiero tiene como objetivo canalizar el dinero de las personas, lo cual permite el 
desarrollo económico y logra que los fondos lleguen desde aquellos individuos que disponen de recursos monetarios, a quienes carecen de los mismos. El sistema financiero ecuatoriano está constituido por: el sistema público, el privado, y el Popular y Solidario (Asamblea Nacional de la República del Ecuador, 2014).

La estructura de cada mercado se identifica por los ambientes o características que determinan el tipo de comportamiento que deben tener las empresas y los resultados a obtener en un futuro. Dentro de las características que decretan la estructura están: el número de oferentes y demandantes, el poder de mercado, grado de concentración y la facilidad o dificultad de entrada de nuevos competidores a la industria. La relación y diferenciación entre estos aspectos dan lugar a la existencia de diferentes estructuras o tipos de mercados (Cuellar-Río, 2007).

Al referirnos a concentración de mercado es importante conocer que se refiere al nivel de participación que posee cada empresa dentro de un sector; así Curry y George (1983) definen este concepto como la distribución del tamaño de las organizaciones que venden un determinado producto o productos, de manera similar, Obando (2015) menciona que se conforma por el número total de firmas presentes en determinado grupo de organizaciones. Para Rodríguez (2002) como se cita en Castañeda (2007) se refiere a la estructura de la industria, entendiendo esta como el número de empresas y la forma en la que participan en el mismo.

Según Sanchis y Mañez (2002), concentración se refiere al grado en el cual la producción de un sector industrial se encuentra concentrado en manos de un reducido grupo de grandes empresas; a su vez, Flor (2003) indica que existen medidas que proporcionan información acerca del grado de uniformidad en el que se reparte el mercado entre sus oferentes. Para Zurita (2014) y acorde a estudios realizados por varios autores como Hannah y Kay, Curry y George, Hall y Tideman, los indicadores del grado de concentración deben cumplir una serie de criterios, dentro de los cuales los principales son: ser fáciles de calcular e interpretar, poder ser calculados a partir de una sola variable y debe ser independiente del tamaño absoluto del mercado analizado. La medición de la participación de cada firma se realiza en base a diferentes tipos de indicadores, con los cuales se determina la existencia o no de poder. La concentración se presenta en todos los mercados alrededor del mundo, generando una lucha constante entre los organismos de control y las empresas; eventualidades que se dan con el objetivo de velar por los intereses de la población (Morales, Cordova, Altamirano, y Lema, 2018).

Castañeda (2007), manifiesta que la utilización de indicadores en el estudio de la concentración representa un papel fundamental para el análisis de poder de mercado, con el principal objetivo de precisar si esta refuerza una posición de dominio y si permite obtener efectos anticompetitivos. A su vez, Segarra (2001) indica que la utilización de estos indicadores permite conocer si un mercado se encuentra cerca de una situación de competencia o de monopolio.

Por otra parte Morales, et al., (2018) indican que en un mercado de oligopolio la concentración tiene una relación directa con el margen de utilidad de las empresas, esto es evidenciado por (Manzano, 2017). En definitiva, las medidas utilizadas tienden a determinar la importancia que tienen las empresas de un mercado, y observar si un grupo de estas ejercen poder sobre los precios y cantidades ofertadas.

La utilización de indicadores de concentración suele ser de gran utilidad al momento de medir o analizar los posibles efectos de fusión de organizaciones y el nivel de competitividad que estas pueden tener; de igual manera permiten realizar un análisis de barreras ante el ingreso de nuevas empresas. La existencia de agencias encargadas de medir los efectos de concentración y fusión centran sus estudios en el análisis de las cuotas de participación, pudiendo ser tomadas con datos actuales o proyectados (Federal Trade Commission, 1997). Estos indicadores también sirven para el análisis de eficiencia de las empresas, ligado al tamaño de las mismas y la cobertura de mercado que tengan; debido a que un mayor tamaño y menor concentración de mercado resulta útil en la búsqueda de ventajas competitivas (De Jorge Moreno y Díaz, 2018).

Si bien el Indice $\mathrm{HHI}$ fue desarrollado para la economía estadounidense, su aplicabilidad en los diferentes mercados a nivel mundial, su aplicabilidad ha sido evidenciada por diferentes autores, no obstante, la premisa de su creación obedece a la necesidad de las autoridades de defensa de la competencia, quienes buscaban determinar si el nivel de competencia en un mercado de cualquier sector económico es adecuado. Así, su utilización se respalda en las economías que dispongan de organismos de control. De manera similar, su aplicabilidad se sustenta en la existencia de las variables de análisis, mismas que por el tipo de formulación matemática que disponen, no generan variaciones que vayan ligadas a factores externos de índole económico o político.

Barbecho y Puchi (2018) citan el trabajo realizado por Castro (2016) e indican que entre las metodologías más utilizadas en la medición de la relación entre concentración y competencia se sitúan dos tipos: los indicadores estructurales y los no estructurales; dentro de los primeros las técnicas más utilizadas son el Índice de Concentración (CR), Índice de Herfindahl-Hirschman (HHI), y dentro de las segundas el índice de Lerner y el modelo de Panzar y Rosse.

Desde otro punto de vista, Zurita (2014) manifiesta que, tras la revisión de diferentes estudios, se evidencia que los efectos de concentración sobre competencia son poco contrastados, debido a que, en sistemas bancarios completos, no depende de la simple distinción entre bancos grandes y pequeños; además concluyen que no hay una relación directa entre mayor concentración y poder de mercado. Por otro lado, en el caso de países 
desarrollados y bancos muy grandes, el grado de competencia es menor al incrementar la concentración. También, se debe tener presente que al estudiar la competencia existen muchas más variables que afectan directamente a la actividad de las entidades y el nivel de competencia, entre las principales están: la herencia histórica política de cada país, la disponibilidad del mercado a brindar información, el ciclo económico, el entorno institucional, entre otras.

\section{Estado del arte}

La revisión literaria permite evidenciar que existen diferentes investigaciones en el ámbito local e internacional en las cuales se recalca la importancia de este tipo de análisis y su rendimiento en los sistemas financieros de cada país; los trabajos consultados y el aporte que brinda cada uno al estudio de esta problemática en los mercados a nivel latinoamericano se describe a continuación.

Martín, Dominguez, Perea, Saca, y Sánchez (2011) determianron que existe una relación entre la concentración bancaria y la competitividad del mercado de capitales; además, altas tasas de concentración disminuyen la competitividad; en su estudio observaron que países como Perú, Ecuador y Argentina obtuvieron mayores ratios con el indice CR1, por otra parte Perú, Ecuador y Chile con el CR3, finalmente el índice $\mathrm{HHI}$ indico que Brasil es el país con mayor nivel de concentración.

Ames, Manrique, Portocarrero y Ventura (2015) evidenciaron que en el mercado bancario peruano posee un alto nivel de concentración; además, que las tasas de interés en comparación con otros países de la región son más altas. También se encontró que más del $50 \%$ de la cuota de mercado en Perú se encuentra dividida únicamente en los dos bancos más grandes. Situación contraria se observa en el mercado bancario argentino, en donde los principales resultados indican que este sistema posee condiciones de competencia monopolística y que su estructura presentó un incremento de la concentración y de la desigualdad de tamaño entre grandes y pequeñas entidades, así como una mayor participación de la banca pública ( Pajón, 2016).

En centroamérica, un estudio realizado en México obtuvo como principales resultados que existe una disminución de los índices de concentración y por lo tanto un incremento en la competencia, además que el resultado alcanzado en el enfoque de poder de mercado nos indica la existencia de competencia monopolística (Gómez, Ríos, y Zambrano, 2018). Por otra parte, un estudio realizado en el sistema bancario colombiano mostró que este presenta una concentración moderada y una estructura de competencia monopolista (Ramírez, 2016).

Flor (2013) determina que el mercado bancario ecuatoriano es moderadamente concentrado y no ejerce ningún grado de poder; por otra parte, Camino, Uzcátegui, y Moran (2017) en un estudio realizado contrastan los hallazgos de Flor, encontrando que el mercado bancario en Ecuador posee una estructura oligopolista y una concentración moderada; además, no se descarta la opción, que al darse una fusión de los cuatro bancos más grandes, estos puedan ejercer cierto poder. Si embargo, Barbecho y Puchi (2018) indican la existencia de una estructura de capital optima en el nivel de competencia, además la existencia de estructura de competencia monopolística y un nivel de concentración moderada.

\section{Descripción del sector de análisis}

El sector financiero puede ser considerado como el grupo de empresas o instituciones cuyo objetivo común es actuar como intermediarios cambiarios; en otros términos, desarrollan un proceso de colocaciones y prestaciones de dinero para el beneficio de los individuos de una población, en donde una gran cantidad de personas generan ahorro, permitiendo a las organizaciones brindar créditos a quienes requieran el recurso monetario. Se encuentra conformado por diferentes tipos de instituciones, siendo el principal el grupo bancario, el cual históricamente sirve de base en la economía de un país mediante la presencia de Bancos Centrales, considerados el motor del sistema financiero nacional debido a que actúan como emisores de moneda, delimitan y controlan actividades, resguardan el tesoro nacional y la reservas para emisión de dinero circulante.

Sealey y Lindley (1977) plantean que las instituciones financieras centran su giro en dos enfoques: producción e intermediación, el primero considera a los bancos como entidades productoras de depósitos y préstamos, mediante la utilización de insumos como capital y mano de obra; a su vez, el enfoque de intermediación plantea que los bancos actúan como intermediarios que transfieren recursos monetarios mediante préstamos e inversiones desde los agentes excedentes hacia los que presentan déficit, utilizando insumos como: depósitos, otros fondos, equidad y trabajo.

El sistema financiero ecuatoriano en su estructura actual no es ajeno a las definiciones anteriormente expuestas y sus actividades de igual manera; debido a que se encuentra constituido por instituciones públicas y privadas, las cuales están reguladas por organismos públicos como la Superintendencia de Bancos, la Superintendencia de Economía Popular y Solidaria (SEPS) y el Banco Central del Ecuador (BCE). En base a lo mencionado cabe indicar que el estudio se centra en evaluar y comparar organizaciones similares, es decir, quienes pertenecen al mismo subsector, siendo la banca pública y privada.

La información utilizada fue tomada del sitio web de la Superintendencia de Bancos del Ecuador, en este espacio se obtuvo datos de los estados financieros de cierre mensual y anual; los datos considerados para el estudio son las cuentas de capital, cartera, depósitos y colocaciones, además las unidades de análisis están clasificadas en base a su tamaño -grandes, medianos y pequeños-. De manera posterior, los datos fueron analizados mediante técnicas y metodologías de carácter 
cuantitativo en las cuales se aplica los índices establecidos en el estudio.

\section{Metodología}

La metodología utilizada en el estudio es de carácter mixto (cuantitativa y cualitativa) de corte longitudinal, mediante la utilización de datos con una dimensión temporal (20062018) y una dimensión espacial dada en el análisis de varios bancos. Además, se emplea un análisis descriptivo del comportamiento de las variables de estudio a lo largo del tiempo e índices para medir la concentración de mercado.

Los índices considerados en el estudio son: Índice Herfindahl-Hirschman, Índice de Concentración, Índice de Lerner (IL)y de manera complementaria el Índice de Dominancia (ID); considerando al ID como una técnica de carácter estructural debido a la formulación matemática establecida para la realización de su análisis.

\section{Índice Herfindahl-Hirschman (HHI)}

Este índice representa uno de los más utilizado por las agencias, puesto que permite medir la concentración de mercado a partir de la contribución relativa de cada empresa al sector, además clasifica a los mercados en tres tipos (U.S. Department of Justice y Federal Trade Commission, 1997).

- Tipos de mercados

1. No concentrados: HHI por debajo de 1.500

2. Moderadamente concentrados: $\mathrm{HHI}$ entre 1.500 y 2.500

3. Altamente concentrados: $\mathrm{HHI}$ por encima de 2.500

Formulación matemática:

$$
H H I=\sum_{i=1}^{n}\left[\frac{q i}{Q}\right]^{2}=\sum_{i=1}^{n}\left[S_{i}\right]^{2}
$$

En donde:

$q_{i}=$ Participación individual de cada banco en el mercado bancario

$Q=$ Total de participación del sector

$S_{i}=$ Cuota de mercado de la i-ésima unidad bancaria de análisis

$n=$ Total de entidades de análisis

\section{Índice de Dominancia (ID)}

Conforme con Zurita (2014) el índice de dominancia mide la concentración del mercado a partir de los resultados de cada entidad obtenidos en la aplicación del índice $\mathrm{HHI}$, esto es, a partir de la contribución relativa de cada banco a la industria; los resultados del índice varían entre un valor de 0 y 1 ; cuando alcanza el valor de 1 significa que la institución tiene la capacidad de ejercer dominio sobre las otras entidades. En este contexto se deduce que el valor más alto se alcanzaría en situación de monopolio y mínimo para oligopolio.

Formulación matemática

$$
I D=\sum_{i=1}^{n}\left[h_{i}\right]^{2}
$$

En donde:

$$
h_{i}=\frac{s_{i}^{2}}{H H I}
$$

$h_{i}=$ Proporción de la cuota de mercado en el índice $\mathrm{HHI}$

$S_{i}=$ Cuota de mercado de la i-ésima unidad bancaria de análisis

$n=$ Total de entidades de análisis

$H H I=$ índice de Herfindahl-Hirschman

\section{Índice de concentración (CR)}

Dentro de los índices, uno de los más utilizados es el ratio de concentración (CR), el cual mide el grado o porcentaje de producción de las $r$ empresas más grandes dentro del total de la industria (Monsalve, 2006); habitualmente lo más adecuado es medir participación de las 4,8 o 10 instituciones de mayor tamaño.

\section{Formulación matemática:}

$$
C R=\sum_{i=1}^{r} \frac{q i}{Q}=\sum_{i=1}^{r} S_{i}
$$

$$
0<C R<1
$$

En donde:

$q_{i}=$ Participación individual de cada empresa en el mercado

$Q=$ Total de participación del mercado

$S_{i}=$ Cuota de mercado de la i-ésima unidad bancaria de análisis

$r=$ Número de unidades principales a determinar la concentración, del total de $n$ elementos

\section{Índice de Lerner}

Permite determinar la capacidad de control que tiene una institución sobre un mercado o el poder de una empresa y su facilidad para la fijación de precio; además de obtener un criterio mediante el cual un observador externo a la organización puede precisar la capacidad que tiene para realizar modificaciones a sus precios sin otra 
consideración que el efecto en la demanda y costos de producción.

Formulación matemática

$$
L=\frac{P-c m g}{P}
$$

En donde:

$$
\begin{aligned}
& P=\text { Precio de mercado } \\
& \mathrm{cmg}=\text { Costo marginal }
\end{aligned}
$$

Este índice nos permite determinar las diferentes situaciones (Martínez et al., 2016).

a) Monopolio. - en el caso de monopolio o en la existencia de un cártel, el índice Lerner es igual a $1 / n$.

b) Competencia perfecta. - en este caso el índice de Lerner es igual a cero $(p=c m g)$.

c) Oligopolio. - en el caso de oligopolio el índice de Lerner ponderado para toda la industria es igual a:

$$
L=\frac{P-c m}{P}=\frac{H H I}{n}
$$

$H H I=$ índice de Herfindahl-Hirschman

En donde $\mathrm{cm}$ es el costo marginal ponderado de todas las empresas y $\mathrm{HHI}$ es el índice de concentración del mercado, considerado una variable exógena.

\section{Resultados}

A continuación, se presenta los resultados obtenidos tras la aplicación de los índices: Herfindahl-Hirschman, índice de concentración e índice de dominancia.

Tabla 1. Índice Herfindahl-Hirschman (HHI)

\begin{tabular}{lllll}
\hline Año & Inversiones & Cartera & Depósitos & Capital \\
\hline 2006 & $1.309,50$ & 986,27 & $1.141,58$ & 796,65 \\
\hline 2007 & $1.206,82$ & $1.032,78$ & $1.216,75$ & 695,50 \\
\hline 2008 & $1.091,31$ & $1.130,77$ & $1.226,89$ & 862,61 \\
\hline 2009 & $1.047,46$ & $1.031,73$ & $1.119,29$ & 930,13 \\
\hline 2010 & $1.071,11$ & $1.039,89$ & $1.120,79$ & 940,23 \\
\hline 2011 & $1.212,44$ & 985,11 & $1.133,75$ & 936,65 \\
\hline 2012 & 982,98 & $1.021,97$ & $1.099,48$ & 935,00 \\
\hline 2013 & 954,43 & $1.129,53$ & $1.159,31$ & 813,41 \\
\hline 2014 & $1.318,10$ & $1.136,59$ & $1.214,69$ & 969,31 \\
\hline 2015 & $1.049,39$ & $1.115,64$ & $1.225,14$ & $1.004,52$ \\
\hline 2016 & $1.065,63$ & $1.201,04$ & $1.242,47$ & 971,60 \\
\hline 2017 & 984,88 & $1.063,46$ & $1.170,13$ & 910,78 \\
\hline 2018 & 638,45 & 304,39 & 325,74 & 334,91 \\
\hline Promedio & $1.071,73$ & $1.013,78$ & $1.107,38$ & 853,95 \\
\hline & & & & \\
\hline & & & & \\
& & & & \\
\hline
\end{tabular}

Tabla 2. Índice de Dominancia

\begin{tabular}{lllll}
\hline Año & Inversiones & Cartera & Depósitos & Capital \\
\hline 2006 & 0,000022 & 0,000034 & 0,000031 & 0,000014 \\
\hline 2007 & 0,000022 & 0,000040 & 0,000035 & 0,000019 \\
\hline 2008 & 0,000017 & 0,000044 & 0,000036 & 0,000017 \\
\hline 2009 & 0,000018 & 0,000036 & 0,000035 & 0,000020 \\
\hline 2010 & 0,000024 & 0,000035 & 0,000034 & 0,000019 \\
\hline 2011 & 0,000034 & 0,000032 & 0,000035 & 0,000018 \\
\hline 2012 & 0,000018 & 0,000034 & 0,000034 & 0,000018 \\
\hline 2013 & 0,000035 & 0,000030 & 0,000030 & 0,000020 \\
\hline 2014 & 0,000033 & 0,000034 & 0,000034 & 0,000018 \\
\hline 2015 & 0,000018 & 0,000030 & 0,000034 & 0,000018 \\
\hline 2016 & 0,000024 & 0,000035 & 0,000033 & 0,000018 \\
\hline 2017 & 0,000019 & 0,000029 & 0,000032 & 0,000017 \\
\hline 2018 & 0,000979 & 0,005180 & 0,004902 & 0,002785 \\
\hline Promedio & 0,000097 & 0,000430 & 0,000408 & 0,000231 \\
\hline
\end{tabular}

Fuente: Elaboración propia a partir de Superintedencia de Bancos (2019a)

Las tablas 1 y 2 muestran respectivamente los resultados obtenidos en los índices HHI e ID, permiten inferir que la banca ecuatoriana presenta un mercado no concentrado, dicho de otra forma, no se evidencia una estructura monopolística, debido a que no existe una institución que domine a las demás; más bien, presenta características de tipo oligopolista, que se verifican en los ratios obtenidos del índice $\mathrm{HHI}$ ubicados en un valor menor a 1.500, además el ID presenta valores entre 0 y 1 ; finalmente este resultado se complementa con la presencia de más de 25

\begin{tabular}{|c|c|c|c|c|}
\hline Año & INVERSIONES & CARTERA & DEPÓSITOS & CAPITAL \\
\hline 2006 & 68,91 & 49,62 & 57,33 & 35,80 \\
\hline 2007 & 65,06 & 50,09 & 57,91 & 37,18 \\
\hline 2008 & 57,43 & 50,81 & 58,43 & 34,89 \\
\hline 2009 & 55,56 & 48,49 & 55,25 & 34,48 \\
\hline 2010 & 52,85 & 48,86 & 54,92 & 37,69 \\
\hline 2011 & 59,25 & 48,93 & 55,76 & 38,89 \\
\hline 2012 & 40,61 & 50,30 & 53,53 & 40,92 \\
\hline 2013 & 38,61 & 41,38 & 42,28 & 36,04 \\
\hline 2014 & 50,23 & 54,89 & 57,06 & 41,80 \\
\hline 2015 & 47,07 & 54,66 & 57,49 & 43,24 \\
\hline 2016 & 47,98 & 56,24 & 58,40 & 42,60 \\
\hline 2017 & 45,02 & 53,35 & 55,97 & 40,14 \\
\hline 2018 & 25,71 & 28,86 & 29,84 & 24,97 \\
\hline Promedio & 50,33 & 48,96 & 53,40 & 37,59 \\
\hline
\end{tabular}
instituciones bancarias en cada año de análisis, mostrando ser un sector con varios oferentes.

Tabla 3. Índice de Concentración Bancos Privados Grandes 
Tabla 4. Índice de Concentración Bancos Públicos

\begin{tabular}{lllll}
\hline Año & Inversiones & Cartera & Depósitos & Capital \\
\hline 2006 & 7,58 & 10,00 & 3,58 & 35,94 \\
2007 & 7,98 & 11,38 & 3,57 & 32,99 \\
2008 & 7,82 & 13,55 & 4,19 & 37,86 \\
2009 & 14,98 & 18,64 & 10,57 & 39,21 \\
2010 & 17,06 & 20,09 & 11,11 & 36,73 \\
2011 & 12,99 & 18,47 & 10,71 & 36,21 \\
2012 & 34,62 & 17,58 & 13,92 & 34,52 \\
2013 & 30,42 & 13,78 & 11,48 & 29,02 \\
2014 & 30,47 & 16,06 & 12,83 & 35,31 \\
2015 & 30,80 & 16,71 & 12,58 & 33,34 \\
2016 & 25,77 & 16,13 & 11,48 & 33,12 \\
2017 & 27,30 & 14,94 & 11,59 & 32,20 \\
2018 & 12,49 & 8,12 & 5,79 & 18,78 \\
Promedio & 20,02 & 15,03 & 9,49 & 33,48 \\
\hline
\end{tabular}

Fuente: Elaboración propia a partir de Superintedencia de Bancos (2019a)

Los resultados presentes en las tablas 3 y 4 , indican que tras la aplicación del índice CR los bancos privados más grandes del país tienen mayor presencia en el mercado que la banca pública; sin embargo es notorio que a lo largo del periodo las instituciones públicas han tenido un notable crecimiento, lo cual se puede evidenciar haciendo una comparación entre los años 2006 y 2018 en la cuenta de inversiones, en la cual, al año 2006 la banca privada presenta una concentración del $68,91 \%$ frente al $7,58 \%$ del sector público, mientras que en el 2018 , esta situación cambia notablemente al presentar índices del 25,71\% y $12,45 \%$ respectivamente. Casos similares se pueden observar en las cuentas de cartera y depósitos.

Tabla 5. Índice de Lerner

\begin{tabular}{|l|l}
\hline Año & Ratio \\
\hline 2006 & 0,94 \\
\hline 2007 & 0,94 \\
\hline 2008 & 0,97 \\
2009 & 0,94 \\
2010 & 0,95 \\
2011 & 0,95 \\
2012 & 0,95 \\
2013 & 0,93 \\
2014 & 0,95 \\
2015 & 0,95 \\
2016 & 0,95 \\
2017 & 0,95 \\
2018 & 0,89 \\
Promedio & 0,94 \\
\hline
\end{tabular}

Fuente: Elaboración propia a partir de Superintedencia de Bancos (2019a)

Los resultados obtenidos mediante la aplicación del índice de Lerner (tabla 5), nos permiten tener una aproximación a la realidad del sector y el comportamiento dado entre la fijación de precios frente al costo incurrido por cada institución, evidenciando que los bancos están en condiciones de manejar sus precios; sin embargo, se debe considerar que estas deben oscilar entre los niveles máximos y mínimos esatablecidos por el Banco Central, por ejemplo: las tasas de interes activas y pasivas.

\section{Conclusiones}

En síntesis, la realización del estudio ha permitido tener un acercamiento al nivel de concentración y competencia que tiene el sector bancario ecuatoriano, en este aspecto se toma como punto de partida la revisión de diferentes casos de estudio realizados a nivel país y de economías vecinas, tales como Perú, Colombia, Argentina e incluso a nivel de Centroamérica como México; el análisis de los diferentes trabajos nos permitió evidenciar que entre las metodologías más utilizadas esta la aplicación de los índices: CR, HHI, IL, por ello se considera importante para el estudio utilizar estos; y de manera adicional incluir el índice de dominancia cuyos resultados permiten verificar lo obtenido tras la aplicación del índice $\mathrm{HHI}$.

Tras la aplicación de los diferentes índices en los bancos privados y públicos ecuatorianos en el periodo 2006-2018, se puede afirmar que el estudio cumplió de manera satisfactoria su objetivo principal "Realizar un análisis de concentración en el sistema bancario ecuatoriano y determinar la existencia de algún tipo de poder de mercado"; así, se puede evidenciar en los resultados, que el sector bancario ecuatoriano no presenta concentración de mercado, dado que el índice $\mathrm{HHI}$ muestra datos inferiores a 1500, de manera simultánea el índice de dominancia presenta valores entre 0 y 1 confirmando la inexistencia de concentración. Por otra parte, el índice CR confirma que no es un mercado concentrado, no obstante, entre los cuatro bancos privados grandes y los bancos públicos captan más del $50 \%$ de participación, y a su vez, los bancos grandes muestran mayor participación que las entidades públicas. En consecuencia, los resultados obtenidos por tres índices nos permiten evidenciar que el sistema bancario ecuatoriano es de tipo oligopolio, y su aplicabilidad se justifica en la existencia de entidades que controlan las actividades de cada institución, deduciendo el comportamiento de estas como autoridades de defensa de la competencia.

La estructura del sistema financiero ecuatoriano indica un decremento en la concentración de la banca privada y un incremento en la banca pública, así como desigualdad de tamaño y ratios obtenidos entre grandes, medianas y pequeñas entidades. También se deduce que a nivel individual no existe una entidad que muestre mantener un dominio sobre las demás, situación que resulta contraria si se suman los ratios obtenidos por las unidades en cada grupo de instituciones según su tamaño, por ello no se descarta que los bancos grandes puedan ejercer algún tipo de poder.

Los resultados obtenidos en los índices de concentración CR también indican un incremento de la banca pública frente a la privada, debido a que la brecha diferencial existente entre estos sectores en las cuentas de inversión, cartera y depósitos es menor en los últimos años, situación que resulta diferente en la cuenta de capital. Ante esto se puede acotar que esta situación podría ser el resultado de 
un mejor manejo del sistema financiero público y la recuperación de la confianza por parte de los clientes.

Los resultados del índice de Lerner, al encontrase por encima del $93 \%$ en todos los años de análisis, indican que los bancos en su estado actual, bajo el supuesto de no existir parámetros de control, podrían fijar sus precios tasas de interés, cobros por servicios, entre otros-. También se evidencia que existe un manejo óptimo de costos y un alto margen de utilidad entre precio y costo; cabe mencionar que en el desarrollo de actividades de las instituciones la fijación de precios se encuentra regulada por organismos como: Banco Central, el Ministerio de Finanzas y la Superintendencia de Bancos.

Finalmente, la revisión de literatura nos permite observar que conforme a autores como Barbecho y Puchi (2018), y Flor (2013) el mercado ecuatoriano es moderadamente concentrado y monopolista respectivamente, siendo esto contrario a nuestro hallazgo que confirma lo determinado por Camino et al., (2017) quienes indican que el mercado ecuatoriano posee una estructura oligopolista. Además, se determinó una característica común del sistema bancario con el de otros países, siendo esta la presencia de la banca privada, la cual muestra ser un eje fundamental y mayoritario en el ámbito financiero.

\section{Referencias}

Ames, C., Manrique, L., Portocarrero, M., \& Ventura, L. (2015). Concentraciones Bancarias en el Perú y sus consecuencias: La necesidad del control Ex Ante. Universidad ESAN, Lima.

Asamblea Nacional de la República del Ecuador. (2014). Código orgánico monetario y financiero. Quito. Retrieved from www.registroficial.gob.ec

BanEcuador. (2018). Programa de educación financiera. Quito. Retrieved from https://www.banecuador.fin.ec/wpcontent/uploads/2018/04/Modulo-3-Sistemafinanciero-nacional.pdf

Barbecho, K., \& Puchi, M. (2018). Competencia y estabilidad en el sistema bancario: Evidencia empírica para el Ecuador durante el periodo 2001 2016. Universidad de Cuenca, Cuenca.

BCE. (2018). Cuentas Nacionales Trimestrales. Retrieved from

https://contenido.bce.fin.ec/home1/estadisticas/cntri mestral/CNTrimestral.jsp

Camino, S., Uzcátegui, C., \& Moran, J. (2017). Estructura de mercado del sistema bancario ecuatoriano: concentración y poder de mercado. Cumbres, 4(1), 9-16.

Castañeda, C. C. (2007). Indicadores de concentración: una revisión del marco conceptual y la experiencia internacional. Ciencias de Relaciones Empresariales, 1-46. Retrieved from https://www.osiptel.gob.pe/Archivos/info_empresas/P oliticas_competencias/DocumentoTrabajo002-GRE2007Concentracion2.pdf

Cuellar-Río, M. (2007). Estructura de mercado y eficiencia. Temas de Ciencia y Tecnología, 11(33), 3-12. Retrieved from http://www.utm.mx/ temas/temas- docs/ensayo1t33.pdf

Curry, B., \& George, K. D. (1983). The Journal of Industrial Economics. The Journal of Industrial Economics, 31(3), 203-255.

De Jorge Moreno, J., \& Díaz, J. (2018). Análisis de la productividad, eficiencia y sus factores explicativos: el caso de las empresas colombianas, 2005-2010. Revista de Métodos Cuantitativos Para La Economía y La Empresa, (26), 315-343.

Federal Trade Commission. (1997). Horizontal Merger Guidelines. Review of Industrial Organization, 8(2), 231-256. https://doi.org/10.1007/BF01034180

Flor, G. (2013). Análisis de concentración, poder de mercado y de la incidencia de la regulacion de tasas de interes en los productos de credito del sector bancario ecuatoriano en el periodo 2003-2011. Pontificia Universidad Católica del Ecuador.

Gómez, T., Ríos, H., \& Zambrano, A. (2018). Competencia y estructura de mercado del sector bancario en México. Contaduría y Administración, 63(1), 1-22.

Manzano, D. (2017). Rentabilidad , solvencia y concentración del sector seguros español. Cuadernos de Información Económica, (262), 41-48.

Martín, M., Dominguez, J., Perea, J., Saca, F., \& Sánchez, S. (2011). La Concentración Bancaria y su Impacto en los Mercados de Capitales de los Países Emergentes. Anales de Estudios Económicos y Empresariales, XXI, 159-177.

Martínez, A., Zuleta, L., Misas, M., \& Jaramillo, L. (2016). La competencia y la eficiencia en la banca colombiana. (La Imprenta Editores, Ed.) (Primera). Bogota: FEDESARROLLO Retrieved from http://hdl.handle.net/11445/3298

Monsalve, F. (2006). Introducción a la Economía Industrial. Retrieved from https://studylib.es/doc/5247327/tema1.-introducción-a-la-economía-industrial

Morales, F. J., García, G., \& Ángeles, E. (2013). Sistema financiero y actividad económica en México : negocio y divergencia del sector bancario. Análisis Económico, XXVII(67), 172-198. Retrieved from http://www.redalyc.org/html/413/41329570010/

Morales, L., Cordova, A., Altamirano, L., \& Lema, E. (2018). ¿Son rentables las empresas concentradas? El caso del sector de curtido de pieles en el Ecuador. Retos, 8(15), 153-166 https://doi.org/10.17163/ret.n15.2018.10

Obando, C. (2015). Supermercados en Ecuador: oligopolios e implicaciones de la Ley Orgánica de Regulación y Control del Poder de Mercado. Pontificia Universidad Católica del Ecuador.

Pajón, I. (2016). Evaluación de la competencia del sistema bancario argentino periodo 2006- 2016 mediante un modelo de Panzar-Rosse.

Penrose, E. (1958). Teoría del crecimiento de la Empresa.

Ramírez, C. (2016). Relación entre concentración, competencia y estabilidad en el sector bancario colombiano (2005-2014). Universidad del Valle.

Romero, B. (2015). La Estructura del Sistema Financiero Ecuatoriano. Retrieved from https://tusfinanzas.ec/laestructura-del-sistema-financiero-ecuatoriano/

Sanchis, A., \& Mañez, J. (2002). Estructura de Mercado y Concentración. 
Sealey, C., \& Lindley, J. (1977). Inputs, outputs, and a theory of production and cost at depository financial institutions. Journal of Finance, 4(32), 1251-1266.

Segarra, A. (2001). Mercados y empresas, 25-73.

Superintedencia de Bancos (2019a). Boletines estadísticos. Retrieved from http://oidprd.sbs.gob.ec/practg/sbs_index?vp_art_id= $5036 \& v p \_t i p=2 \& v p \_b u s c r=41$

Superintedencia de Bancos. (2019b). Superintendencia de Bancos. Retrieved from http://oidprd.sbs.gob.ec/practg/p_index

Tarziján, J., \& Paredes, R. (2006). Organización industrial para la estrategia empresarial. (Pearson Educación, Ed.) (Segunda). México: Pearson Educación. Retrieved https://eva.fcs.edu.uy/pluginfile.php/58227/mod_reso urce/content/1/Organización Industrial 2edi Tarziján.pdf

Vargas, G., \& Rodríguez, C. (2016). Oligopolio y estrategias de competencia en el mercado de minoristas en México. Economía Informa, 400, 3-23. https://doi.org/10.1016/j.ecin.2016.09.002

Zurita, J. (2014). Análisis de la concentración y competencia en el sector bancario. Madrid. 